\title{
An Extension of the Poincar'e Lemma of Differential Forms
}

\author{
Zhaoyang Tang, Jianmin Zhu, Jianhua Huang, Jin Li \\ Department of Mathematics and System Science, National University of Defense Technology, \\ Changsha, China \\ Email: tzymath@gmail.com
}

Received October 30, 2012; revised November 30, 2012; accepted December 7, 2012

\begin{abstract}
This paper is to extend the Poincar'e Lemma for differential forms in a bounded, convex domain [1] in $R^{n}$ to a more general domain that, we call, is deformable to every point in itself. Then we extend the homotopy operator $T$ in [1] to the domain defromed to every point of itself.
\end{abstract}

Keywords: Differential Forms; Poincar'e Lemma; Domain Deformed to Every Point

\section{Introduction}

In [2], we have the Converse of the Poincar'e Lemma:

Lemma 1.1. Let $U$ be a domain in $R^{n}$ which can be deformed to a point $P$. Let $\omega$ be a $(p+1)$-form on $U$ such that $d \omega=0$. Then there is a $p$-form $\alpha$ in $U$ such that

$$
\omega=\mathrm{d} \alpha .
$$

And in [1] we have

Lemma 1.2. Let $D$ be a bounded, convex domain in $R^{n}$. To each $y \in D$ there corresponds a linear operator $K_{y}: C^{\infty}\left(D, \Lambda^{l}\right) \rightarrow C^{\infty}\left(D, \Lambda^{l}\right)$ defined by

$$
\begin{aligned}
& \left(K_{y} \omega\right)\left(x ; \xi_{1}, \cdots, \xi_{l-1}\right) \\
& =\int_{0}^{1} t^{l-1} \omega\left(t x+y-t y ; x-y, \xi_{1}, \cdots, \xi_{l-1}\right) \mathrm{d} t
\end{aligned}
$$

and the decomposition

$$
\omega=\mathrm{d}\left(K_{y} \omega\right)+K_{y}(\mathrm{~d} \omega)
$$

holds at any point $y$ in $D$.

In this paper, we extend the results of both of them. First we extend the bounded, convex domain $D$ to the domain that deformed to every interior point. Then we not only gain that the closed form is the exact form, but every form can be decomposited to two parts where one of them is an exact form and another is a form related to the exterior differential of the form.

\section{Preliminaries}

It's well-known that differential forms are the generalizations of the functions and have been applied to many fields such as potential theory, partial differential equa- tions, quasiconformal mappings, nonlinear analysis, electromagnetism and control theory. First, we introduce some notations and preliminaries about differential forms. Let $U$ denote an open subset of $R^{n}, n \geq 2$ and $R=R^{1}$. Let $\left\{e_{1}, e_{2}, \cdots, e_{n}\right\}$ denote the standard orthogonal basis of $R^{n} . \Lambda^{l}\left(R^{n}\right)$ is the linear space of $l$-covectors, generated by the exterior products $e_{I}=e_{i_{1}} \wedge e_{i_{2}} \wedge \cdots \wedge e_{i_{l}}$, corresponding to all ordered $l$-tuples

$I=\left(i_{1}, i_{2}, \cdots, i_{l}\right), 1 \leq i_{1}<i_{2}<\cdots<i_{l} \leq n, l=0,1, \cdots, n$. The Grassman algebra $\Lambda=\Lambda\left(R^{n}\right)=\oplus_{l=0}^{n} \Lambda^{l}$ is a graded algebra with respect to the exterior products.

A differential $l$-form $\omega$ on $U$ is a Schwartz distribution on $U$ with values in $\Lambda\left(R^{n}\right)$. Let $D^{\prime}\left(U, \Lambda^{l}\right)$ denote the space of all differential $l$-forms and the class of infinitely differentiable $l$-forms on $U$ by $C^{\infty}\left(U, \Lambda^{l}\right)$.

Then we define the mapping $f^{*}$ for a smooth mapping $f$ on $U$ into $V$, where $U$ is a domain in $R^{m}$ and $V$ is a domain in $R^{n}$, that is

$$
f: U \rightarrow V
$$

We denote by $x_{1}, \cdots, x_{m}$ the coordinates of $R^{m}$ and by $y_{1}, \cdots, y_{n}$ the coordinates of $R^{n}$. Then we can write

$$
y_{i}=y_{i}\left(x_{1}, \cdots, x_{m}\right)
$$

to show that the point with coordinates $x$ is transformed by $f$ to the point with coordinated $y$. The function $y_{i}(x)$ are smooth. Now we define the map $f^{*}$ taking $l$-forms on $V$ to $l$-forms on $U$ :

$$
f^{*} \Lambda^{l}(V) \rightarrow \Lambda^{l}(U) .
$$

And there are basic properties for the mapping $f^{*}$ we'll use in the following statement. 
Lemma 2.1. If $\omega$ is a $l$-form on $V$, then

$$
d\left(f^{*} \omega\right)=f^{*}(d \omega) .
$$

Lemma 2.2. If $f: U \rightarrow V$ and $g: V \rightarrow W$, then

$$
(f \circ g)^{*}=f^{*} \circ g^{*}
$$

More essential properties for $f^{*}$ can be found in [3]. More preliminaries of differential forms and their applications can be found in [1-15].

Then we define another important mapping:

Definition 2.1. Given a function $\varphi=\varphi(x, u)$ is a continuous for $(x, u)$ [3]. We call a domain $U$ is deformable to a point $p$ if there exists $I \times U \rightarrow U$ such that

$$
\begin{gathered}
\varphi(1, x)=x, \\
\varphi(0, x)=P .
\end{gathered}
$$

Then we can analogously define that a domain is deformable to any point $y \in U$, and denote the function $\varphi_{y}$ as $\varphi$ for every $y$.

\section{Main Results and Proofs}

First, we introduce the "cylinder construction". Let $U$ be a domain in $R^{n}$ that is deformable to any $y \in U$ just like we have defined. We denote by $[0,1]$ the unit interval on the $t$-axis and consider the cylinder or product space $I \times U$.

This consists of all pairs $(t, x)$ where $0 \leq t \leq 1$ and $x$ runs over points of $U$. We point out the two maps which identify $U$ with the top and bottom of the cylinder, that is

$$
\begin{aligned}
& j_{1}: U \rightarrow I \times U, j_{1}(x)=(1, x), \\
& j_{0}: U \rightarrow I \times U, j_{0}(x)=(0, x) .
\end{aligned}
$$

Thus

$$
j_{1}^{*}: F^{p}(I \times U) \rightarrow F^{p}(U) \quad(i=0,1) .
$$

For example, to form $j_{1}^{*} \omega$ where $\omega$ is a form on $I \times U$, we simply replace $t$ by 1 wherever it occurs in $\omega$ (and $\mathrm{d} t$ by 0 correspondingly). Now we form a new operation $K_{y}$ for any $y \in U$,

$$
K_{y}: F^{p+1}(I \times U) \rightarrow F^{p}(U)
$$

$K_{y}$ is defined on monomials by the formulas:

$$
\begin{gathered}
K_{y}\left(a(t, x) \mathrm{d} x_{I}\right)=0 \\
K_{y}\left(a(t, x) \mathrm{d} t \mathrm{~d} x_{J}\right)=\int_{0}^{1}(a(t, x) \mathrm{d} t) \mathrm{d} x_{J},
\end{gathered}
$$

and on general differential forms by summing the results on the monomial parts. Here is the basic property of $K_{y}$ :

Lemma 3.1. If $\omega$ is any $(p+1)$-form on $I \times U$, then

$$
K_{y}(d \omega)+d\left(K_{y} \omega\right)=j_{1}^{*} \omega-j_{0}^{*} \omega .
$$

Proof: We only need to check this for monomials.

Case 1. $\omega=a(t, x) \mathrm{d} x_{H}$.

We have $K_{y} \omega=0, \mathrm{~d} K_{y} \omega=0$,

$$
\begin{gathered}
\mathrm{d} \omega=\frac{\partial a}{\partial t} \mathrm{~d} t \mathrm{~d} x_{H}+[\text { terms free of } \mathrm{d} t] \\
K_{y} \mathrm{~d} \omega=\left(\int_{0}^{1} \frac{\partial a}{\partial t} \mathrm{~d} t \mathrm{~d} x_{H}=[a(1, x)-a(0, x)] \mathrm{d} x_{H}\right)
\end{gathered}
$$

But $j_{1}^{*} \omega=a(1, x) \mathrm{d} x_{H}, j_{0}^{*} \omega=a(0, x) \mathrm{d} x_{H}$. So the formula is valid .

Case 2. $\omega=a(t, x) \mathrm{d} t \mathrm{~d} x_{J}$.

First notice $j_{1}^{*} \omega=j_{0}^{*} \omega=0$. Next we have

$$
\begin{aligned}
K_{y} \mathrm{~d} \omega & =K_{y}\left[-\sum \frac{\partial a}{\partial x_{i}} \mathrm{~d} t \mathrm{~d} x_{i} \mathrm{~d} x_{J}\right], \\
& =-\sum\left(\int_{0}^{1} \frac{\partial a}{\partial x_{i}} \mathrm{~d} t\right) \mathrm{d} x_{i} \mathrm{~d} x_{J} \\
\mathrm{~d} K_{y} \omega & =\mathrm{d}\left[\left(\int_{0}^{1} a(t, x) \mathrm{d} t\right) \mathrm{d} x_{J}\right] \\
& =\sum \frac{\partial}{\partial x_{i}} \mathrm{~d} t \mathrm{~d} x_{i} \mathrm{~d} x_{J}
\end{aligned}
$$

So the formula works, again.

We can easily get the following conclusion:

Lemma 3.1. For the mapping $\varphi: I \times U \rightarrow U$, the boundary conditions may be interpreted in terms of the $j_{i}$ as follows:

$$
\varphi_{\circ} j_{1}=I, \varphi_{\circ} j_{0}=y,
$$

if $U$ is deformable to the point $y$.

For an $(l+1)$-form $\omega$ on $U$ we have

$$
j_{1}^{*}[\varphi * \omega]=\omega, j_{0}^{*}[\varphi * \omega]=0 .
$$

Now we state and prove the main result.

Theorem 3.1. Assume $U$ is a domain in $R^{n}$ which can be deformed to every point $y \in U$. Let $\omega$ be an $(l+$ $1)$-form on $U$. Then there is

$$
K_{y}\left[\mathrm{~d}\left(\varphi^{*} \omega\right)\right]+\mathrm{d}\left[K_{y}\left(\varphi^{*} \omega\right)\right]=\omega .
$$

Proof: We only substitute $\varphi^{*} \omega$ in the above formula of Lemma 3.1. And with Equation (22), we finish the proof.

Thus we finish the extension. It's interesting to see if $\mathrm{d} \omega=0$, then $\mathrm{d}\left(\varphi^{*} \omega\right)=\varphi^{*}(\mathrm{~d} \omega)=0$. Hence with the formula above we have $\omega=\mathrm{d} \alpha$ where $\alpha=K_{y}\left(\varphi^{*} \omega\right)$. This is just the generalization of the converse of the Poincar'e Lemma in [3], which shows that closed form is an exact form.

Corollary 3.1. Assume $U$ is a domain in $R^{n}$ which 
can be deformed to every point $y \in U$. If $\omega$ is a closed $(l+1)$-form on $U$, then it is an exact form. Then we can construct a homotopy operator

$T: C^{\infty}\left(U, \Lambda^{l}\right) \rightarrow C^{\infty}\left(U, \Lambda^{l}\right)$ by averaging $K_{y}$ over all points $y \in U$ :

$$
T \omega=\int_{U} \psi(y) K_{y}\left(\phi^{*} \omega\right) \mathrm{d} y
$$

where $\psi$ in $C_{0}^{\infty}(U)$ is normalized so that $\int_{U} \psi(y) \mathrm{d} y=1$. It is obvious that the main result of this article remains valid for the operator $T$ :

$$
\omega=\mathrm{d}(T \omega)+T(\mathrm{~d} \omega) .
$$

We begin with the equation of Lemma 3.1

$$
K_{y}(\mathrm{~d} \omega)+\mathrm{d}\left(K_{y} \omega\right)=j_{1}^{*} \omega-j_{0}^{*} \omega .
$$

Multiplying $\psi(y)$ to it and integraling on $U$, we have

$$
\int_{U} \psi(y) \omega \mathrm{d} y=\int_{U} \psi(y) K_{y}\left[\mathrm{~d}\left(\phi^{*} \omega\right)\right] \mathrm{d} y
$$

Then with $\int_{U} \psi(y) \mathrm{d} y=1$, we obtain

$$
\begin{aligned}
\omega & =\int_{U} \psi(y) K_{y}\left[\phi^{*}(\mathrm{~d} \omega)\right] \mathrm{d} y \\
& +\mathrm{d}\left[\int_{U} \psi(y) K_{y}\left(\phi^{*} \omega\right) \mathrm{d} y\right]
\end{aligned}
$$

which yields the above formula.

\section{Conclusion}

We have obtained an extension of the Poincar'e Lemma for differential forms in a bounded, convex domain in $R^{n}$ to a more general domain. Then we have extended the homotopy operator $T$ to the domain defromed to every point of itself. So all of the conclusions about the homotopy operator $T$ can be extended to the deformed domain.

\section{Acknowledgements}

The research of the author was supported by the Fundamental Research (2010) of NUDT (NO. JC10-02-02).

\section{REFERENCES}

[1] T. Iwaniec and A. Lutoborski, "Integral Estimates for Null Lagrangians," Archive for Rational Mechanics and Analysis, Vol. 125, No. 1, 1993, pp. 25-79. doi:10 .1007/BF00411477
[2] H. Flanders, "Differential Forms with Applications to the Physical Sciences," Dover Publications, Mineola, New York, 1963.

[3] P. R. Agarwal, S. Ding and C. A. Nolder, "Inequalities for Differential Forms,” Springer, New Mexico, 2009. doi:10.1007/978-0-387-68417-8

[4] M. Spivak, "Calculus on Manifolds," Perseus Books Publishing, New York, 1965.

[5] J. Zhu and J. Li, "Some Priori Estimates about Solutions to Nonhomogeneous A-Harmonic Equations," Journal of Inequalities and Applications, Vol. 2010, No. 520240, 2010, Article ID: 520240.

[6] S. S. Ding and J. M. Zhu, "Poincar-Type Inequalities for the Homotopy Operator with $\mathrm{L} \varphi(\Omega)$-Norms," Nonlinear Analysis: Theory, Methods and Applications, Vol. 74, No. 11, 2011, pp. 3728-3735.

[7] J. Zhu, S. Ding and Z. Tang, "The Reverse Holder and Caccioppoli Type Inequalities for Generalized A-Harmonic Equations," Under Review.

[8] S. Ding, "Two-Weight Caccioppoli Inequalities for Solutions of Nonhomogeneous A-Harmonic Equations on Riemannian Manifolds," Proceedings of the American Mathematical Society, Vol. 132, 2004, pp. 2367-2375. doi:10.1090/S0002-9939-04-07347-2

[9] S. Ding, "Local and Global Norm Comparison Theorems for Solutions to the Nonhomogeneous A-Harmonic Equation," Journal of Mathematical Analysis and Applications, Vol. 335, No. 2, 2007, pp. 1274-1293. doi:10.1016/j.jmaa.2007.02.048

[10] M. Giaquinta and J. Soucek, "Caccioppoli's Inequality and Legendre-Hadamard Condition," Mathematische Annalen, Vol. 270, No. 1, 1985, pp. 105-107. doi:10.1007/BF01455535

[11] T. Iwaniec and G. Sbordone, "Weak Minima of Variational Integrals," Journal of Reine Angew Math, Vol. 454, 1994, pp. 143-161.

[12] C. A. Nolder, "Hardy-Littlewood Theorems for A-Harmonic Tensors," Illinois Journal of Mathematics, Vol. 43, 1999, pp. 613-631.

[13] C. A. Nolder, "Global Integrability Theorems for A-Harmonic Tensors," Journal of Mathematical Analysis and Applications, Vol. 247, No. 1, 2000, pp. 236-247. doi:10.1006/jmaa.2000.6850

[14] C. A. Nolder, "Conjugate Harmonic Functions and Clifford Algebras," Journal of Mathematical Analysis and Applications, Vol. 302, No. 1, 2005, pp. 137-142. doi:10.1016/j.jmaa.2004.08.008

[15] B. Stroffolini, “On Weakly A-Harmonic Tensors," Studia Mathematica, Vol. 114, 1995, pp. 289-301. 